International Mathematical Forum, Vol. 9, 2014, no. 20, 977 - 980

HIKARI Ltd, www.m-hikari.com

http://dx.doi.org/10.12988/imf.2014.4489

\title{
The Influence of Primitive Subgroups on the Structure of Finite Groups ${ }^{1}$
}

\author{
Huang Jianhong and $\mathrm{Hu} \mathrm{Bin}^{2}$ \\ School of Mathematics and Statistics \\ Jiangsu Normal University \\ Xuzhou 221116, P.R. China
}

Copyright (C) 2014 Huang Jianhong and Hu Bin. This is an open access article distributed under the Creative Commons Attribution License, which permits unrestricted use, distribution, and reproduction in any medium, provided the original work is properly cited.

\begin{abstract}
A subgroup $H$ of a group $G$ is said to be primitive if it is a proper subgroup of the intersection of all subgroups of $G$ containing $H$ as its proper subgroup. In this paper, we will study the influence of primitive subgroups on the structure of finite groups and some new results are obtained.
\end{abstract}

Mathematics Subject Classification: 20D10, 20D15, 20D20

Keywords: Finite group; Primitive subgroup; cyclic subgroup; Structure of groups

\footnotetext{
${ }^{1}$ Research is supported by NNSF Grant of China (grant: 11071229) and the Research Program of Jiangsu Normal University (Grant: 12XLA06).

${ }^{2}$ Corresponding author
} 
Throughout this paper, all groups considered are finite and $G$ denotes a finite group. The terminologies and notations are standard, as in [1].

The generalized concept of maximal subgroups of a group $G$, namely the primitive subgroups, was introduced by Johnson in 1971 [2]. A subgroup $H$ of $G$ is said to be primitive if it is a proper subgroup of the intersection of all subgroups of $G$ containing $H$ as its proper subgroup. It is interesting to note that every group $G$ has a primitive subgroup and that every proper subgroup of $G$ is the intersection of some primitive subgroups of $G$. Since the intersection of all primitive subgroups is the identity subgroup, we can easily see that the class of all primitive subgroups is obviously wider than the class of all maximal subgroups. Thus, one might naturally except that by extending the conditions from maximality to primitivity, we can obtain some stronger conclusions.

Recall that a group is metabelian if its commutator subgroup is abelian[3, $\S 5.1]$. The following result gives a structure of a $p$-group whose all non-cyclic subgroups are primitive.

Theorem 1.1. Suppose that all non-cyclic subgroups of a group $G$ are primitive. If $G$ is a $p$-group for some prime $p$, then $G$ is a cyclic group or an elementary abelian group of order $p^{2}$ or a metabelian group whose Frattini subgroup $\Phi(G)$ is cyclic.

Proof. Since a $p$-group of order $\leq p^{2}$ is either cyclic or an elementary abelian group, we may assume that $|G| \geq p^{3}$. If $G$ has a unique maximal subgroup $M$, then $G$ is cyclic. So we suppose that $G$ has at least two different maximal subgroups $M_{1}, M_{2}$. Obviously, $M_{1} \cap M_{2}$ is not a primitive subgroup of $G$. By hypothesis, $M_{1} \cap M_{2}$ is cyclic. Consequently, $\Phi(G)$ is cyclic. Since $G$ is a p-group, $G / \Phi(G)$ is an abelian group. Hence $G$ is a metabelian group. This completes the proof.

A group $G$ is said to be a minimal non-cyclic group if $G$ is not cyclic but every proper subgroup of $G$ is cyclic[1, §3.4]. Let $\pi(G)$ denote the set of all prime divisors of $|G|$. A group $G$ is said to be bi-primary if $|\pi(G)|=2$. The following theorem gives the structure of a non-primary nilpotent group whose 
all non-cyclic subgroups are primitive.

Theorem 1.2. Suppose that all non-cyclic subgroups of a group $G$ are primitive. If $G$ is a non-primary nilpotent group, then $G$ is a cyclic group or a bi-primary group in which a Sylow subgroup is a minimal non-cyclic group at least.

Proof. If $G$ is a non-primary nilpotent group, then every Sylow subgroup of $G$ is normal in $G$. Suppose that $|\pi(G)| \geq 3$ and $P, Q, R$ are arbitrary Sylow subgroups of $G$. Then $P Q R=P \times Q \times R$. Obviously, $P Q \cap P R=P(Q \cap P R)=$ $P$ and so $P$ is not a primitive subgroup of $G$. By hypothesis, $P$ is cyclic. Using the same argument as above, every Sylow subgroup of $G$ is cyclic. Hence, $G$ is a cyclic group. Therefore, we suppose that $|\pi(G)|=2$ and $G=P \times Q$, where $P$ is a Sylow $p$-subgroup of $G$ and $Q$ is a Sylow $q$-subgroup of $G$. If $P, Q$ are all cyclic subgroups of $G$, then $G$ is cyclic. Hence we assume that $P$ is not cyclic. Let $P_{1}$ be an arbitrary non-identity proper subgroup of $P$. It is easy to see that $P_{1}=P \cap P_{1} Q$ and so $P_{1}$ is not a primitive subgroup of $G$. By hypothesis, $P_{1}$ is cyclic. It follows that $P$ is a minimal non-cyclic group. This completes the proof.

Theorem 1.3. If every primitive subgroup of a group $G$ is permutable with all Sylow subgroups of $G$, then $G$ is supersoluble.

Proof. Let $H$ be an arbitrary primitive subgroup of $G$. Suppose that $p, q$ are different prime divisors of the index $|G: H|$. By hypothesis, there exist a Sylow $p$-subgroup $P$ and a Sylow $q$-subgroup $Q$ of $G$ such that $H P=P H$ and $H Q=Q H$. Obviously, $H$ is a proper subgroup of $H P$ and of $H Q$. Let $N=H P \cap H Q$. Then $N$ is not a primitive subgroup of $G$ and $N=H(P \cap$ $H Q)=H(H P \cap Q)$. Since $|N: H|=|P \cap H Q: H \cap P|=|H P \cap Q: H \cap Q|$, $|N: H|$ divides $|P|$ and $|Q|$. This implies that $N=H$, which contradicts the primitivity of $H$. Therefore, $|G: H|$ is primary. By the arbitrary choice of $H$, we know that every primitive subgroup of $G$ has primary index in $G$. Hence by Johnson's Theorem [2], $G$ is supersoluble. 


\section{References}

[1] W. Guo, The Theory of Class of Groups, Science Press-Kluwer Academic Publishers, Beijing-New-York-Dordrecht-Boston, 2000.

[2] D.L. Johnson, A note on supersoluble groups, Canadian J. Math., 23(1971), 562-564.

[3] D.J.S. Robinson, A Course in the Theory of Groups, Springer-Verlag, New York-Heidelberg-Berlin, 1982.

Received: April 30, 2014 